\title{
ON THE ANALYTIC CONTINUATION OF DIRICHLET SERIES
}

\author{
RONALD SEELING
}

\begin{abstract}
Given a continuable Dirichlet series, having as sequence of exponents $\left\{\lambda_{n}\right\}_{n=1}^{\infty}$, it is shown that any other Dirichlet series can be continued along the same paths away from its half plane of convergence provided that the two series have the same coefficients and that the difference of their exponents is eventually given by a function analytic at infinity evaluated at the $\lambda_{n}$.
\end{abstract}

There are a number of theorems in the literature which give analytic continuation of Dirichlet series (including Taylor series) if one perturbs the coefficients. See Bierbach [1] or, in particular, Ostrowski [2].

Further, there are theorems dealing with continuability of Dirichlet series if one perturbs the exponents. However, these theorems are either negative in character, in that noncontinuability is demonstrated, or growth estimates and other hypotheses are required. See Mandelbrojt [3].

The following theorem provides continuation of a second series provided only that its exponents eventually differ from that of an original continuable series by a function analytic at infinity evaluated at the exponents of the original series.

THEOREM. Let $\left\{\lambda_{n}\right\}_{n=1}^{\infty}$ and $\left\{\mu_{n}\right\}_{n=1}^{\infty}$ be two sequences of strictly increasing, positive real numbers, tending to infinity. Suppose that there exists a real number $R \geqslant 0$ and a function $g$, analytic in $|z|>R$ and at infinity, such that $g\left(\lambda_{n}\right)=\mu_{n}-\lambda_{n}$ for all integers $n>R$.

Let $F(s)=\sum_{n=1}^{\infty} a_{n} e^{-\lambda_{n} s}$ be a convergent Dirichlet series for $\operatorname{Re} s>\sigma_{F}$ $\left(\sigma_{F}<\infty\right)$. Then $G(s)=\sum_{n=1}^{\infty} a_{n} e^{-\mu_{n} s}$ is a convergent Dirichlet series for $\operatorname{Re} s>\sigma_{G}$ where

$$
\sigma_{G}=\sigma_{F}=\limsup _{n \rightarrow \infty} \frac{\log \left|a_{n}\right|}{\lambda_{n}} \text { if } \limsup _{n \rightarrow \infty} \frac{n}{\lambda_{n}}<\infty
$$

while

$\sigma_{G}=\max (0, a)$ where $a=\limsup _{n \rightarrow \infty}\left(\frac{\log \left|\sum_{m \leqslant n} a_{m}\right|}{\lambda_{n}}\right)<\infty$ if $\limsup _{n \rightarrow \infty} \frac{n}{\lambda_{n}}=\infty$.

Further, $G(s)=\sum_{n=1}^{\infty} a_{n} e^{-\mu_{n} s}$ can be analytically continued to all points on any path along which $F$ can be analytically continued.

Received by the editors February 1, 1977.

AMS (MOS) subject classifications (1970). Primary 30A16, 30A14.

Key words and phrases. Dirichlet series, analytic continuation along paths.

() American Mathematical Society 1978 
Proof. We note that $\lim \sup _{n \rightarrow \infty} n / \lambda_{n}=\lim \sup _{n \rightarrow \infty} n / \mu_{n}$ and

$$
\limsup _{n \rightarrow \infty}\left(\frac{\log \left|\sum_{m<n} a_{m}\right|}{\lambda_{n}}\right)=\limsup _{n \rightarrow \infty}\left(\frac{\log \left|\sum_{m<n} a_{m}\right|}{\mu_{n}}\right) .
$$

So the statements about convergence of the series follow from known Dirichlet series results. (See Mandelbrojt [3].)

If $g(\infty) \neq 0$, let $g_{1}(z) \equiv g(z)-g(\infty)$ and $\beta_{n}=\mu_{n}-g(\infty)$. We can then prove the theorem for $\left\{\lambda_{n}\right\}_{n=1}^{\infty},\left\{\beta_{n}\right\}_{n=1}^{\infty}$ with $g_{1}\left(\lambda_{n}\right)=\beta_{n}-\lambda_{n}$ and $g_{1}(\infty)=$ 0 . Since $G(s)=\sum_{n=1}^{\infty} a_{n} e^{-\mu_{n} s}$ converges (can be continued) everywhere $\sum_{n=1}^{\infty} a_{n} e^{-\beta_{n} s}=e^{g(\infty) s} G(s)$ converges (can be continued), we can without loss of generality take $g(\infty)=0$.

Let $K>\max \left\{\sigma_{F}, 0\right\}$ and $\tilde{K}=\{w \in \mathbf{C}:|w|<K\}$ and let $\tilde{R}=\{z \in \mathbf{C}$ : $|z|>R$ or $z=\infty\}$. Define $\phi(w, z)=\exp (-w g(z))-1$, holomorphic for $(w, z) \in \mathbf{C} \times \tilde{R}$. Since $\varphi(0, z)=\varphi(w, \infty)=0$ the expansion of $\varphi$ in $\mathbf{C} \times \tilde{R}$ can be written as

$$
\varphi(w, z)=\sum_{n=1}^{\infty} \sum_{k=1}^{\infty} b_{n k} \frac{w^{k}}{z^{n}} .
$$

Let $\varepsilon>0$ be such that $R-[R]<1-\varepsilon$ and

$$
\begin{aligned}
\Phi(w, z) & =\frac{1}{2 \pi i} \oint_{|t|=R+\varepsilon} \phi(w, t) e^{z t} d t \\
& =\sum_{n=1}^{\infty} \sum_{k=1}^{\infty} \frac{b_{n k}}{(n-1) !} w^{k} z^{n-1},
\end{aligned}
$$

holomorphic for all $(w, z) \in \mathbf{C}^{2}$.

Now, for $w \in \tilde{K}, z \in \mathbf{C}$

$$
\begin{aligned}
|\Phi(w, z)| & \leqslant \frac{2 \pi(R+\varepsilon)}{2 \pi} \sup _{|t|=R+\varepsilon}|\varphi(w, t)|\left|e^{z t}\right| \\
& \leqslant(R+\varepsilon) \sup _{|t|=R+\varepsilon}\left(e^{|g(t) \| w|}+1\right) e^{|z z|} \\
& \leqslant(R+\varepsilon)\left(e^{M(R+\varepsilon, g) K}+1\right) e^{(R+\varepsilon)|z|} \\
& \equiv K^{*} e^{(R+\varepsilon)|z|}
\end{aligned}
$$

for $w \in \tilde{K}, z \in \mathbf{C}$. Also,

$$
\begin{aligned}
\int_{0}^{\infty} \Phi(w, t) e^{-z t} d t & =\sum_{n=1}^{\infty} \sum_{k=1}^{\infty} \frac{b_{n k}}{(n-1) !} w^{k} \int_{0}^{\infty} t^{n-1} e^{-z t} d t \\
& =\sum_{n=1}^{\infty} \sum_{k=1}^{\infty} \frac{b_{n k}}{(n-1) !} w^{k} \frac{(n-1) !}{z^{n}}=\varphi(w, z)
\end{aligned}
$$

for $|z|>R,|w|<K$. Let $M$ be a positive integer with $\lambda_{M} \geqslant[R]+1$. One has that $\sum_{n=M}^{\infty} a_{n} e^{-\lambda_{n} s}$ is uniformly convergent on compact subsets of $\operatorname{Re} s>\sigma_{F}$. Further, $\int_{s}^{\infty} \Phi(w, u-s) e^{-\lambda_{n} u} d u$ is a convergent integral for $\operatorname{Re} s>\sigma_{F}, w \in \tilde{K}$ and $n \geqslant M$. Here the path of integration is from $s$ to $1+\sigma_{F}$ then along the real axis to infinity. This holds since for $w \in \tilde{K}, u$ real 
and sufficiently large, from (3),

$$
\left|\Phi(w, u-s) e^{-\lambda_{n} u}\right|<K^{*} e^{(R+\varepsilon)|u-s|}\left|e^{-\lambda_{n} u}\right|<K^{*} e^{(R+e)|s|} e^{\left(R+\varepsilon-\lambda_{n}\right) u}
$$

while for $n \geqslant M, R+\varepsilon-\lambda_{n} \leqslant R+\varepsilon-\lambda_{M} \leqslant R+\varepsilon-[R]-1<0$. Thus one can interchange integration and summation in the integral below and write for $w \in \tilde{K}, \operatorname{Re} s>\sigma_{F}$

$$
\begin{aligned}
h(w, s) & =\int_{s}^{\infty} \Phi(w, u-s) \sum_{n=M}^{\infty} a_{n} e^{-\lambda_{n} u} d u \\
& =\int_{0}^{\infty} \Phi(w, t) \sum_{n=M}^{\infty} a_{n} e^{-\lambda_{n}(t+s)} d t \\
& =\sum_{n=M}^{\infty} a_{n} e^{-\lambda_{n} s} \int_{0}^{\infty} \Phi(w, t) e^{-\lambda_{n} t} d t \\
& =\sum_{n=M}^{\infty} a_{n} e^{-\lambda_{n} s} \varphi\left(w, \lambda_{n}\right) \\
& =\sum_{n=M}^{\infty} a_{n} e^{-\lambda_{n} s}\left\{e^{-w g\left(\lambda_{n}\right)}-1\right\} .
\end{aligned}
$$

Then in the intersection of $\operatorname{Re} s>\sigma_{f}$ and $\tilde{K}\left(=\left\{s:|s|<K, K>\sigma_{f}\right\}\right)$ from (6),

$$
h(s, s)=\sum_{n=M}^{\infty} a_{n} e^{-\mu_{n} s}-\sum_{n=M}^{\infty} a_{n}^{-\lambda_{n} s} .
$$

Letting $F_{M}(s)=\sum_{n=M}^{\infty} a_{n} e^{-\lambda_{n} s}$ we get for $s \in\left\{s \in \mathrm{C}: \operatorname{Re} s>\sigma_{f}\right\} \cap \tilde{K}$,

$$
\begin{aligned}
G(s) & =\sum_{n=1}^{\infty} a_{n} e^{-\mu_{n} s}=\sum_{n=M}^{\infty} a_{n} e^{-\lambda_{n} s}+\sum_{n=1}^{M-1} a_{n} e^{-\mu_{n} s}+h(s, s) \\
& =F_{M}(s)+\sum_{n=1}^{M-1} a_{n} e^{-\mu_{n} s}+h(s, s) .
\end{aligned}
$$

Since $K$ is arbitrarily large, (6) holds for all $s$ with $\operatorname{Re} s>\sigma_{F}$. Since the function $\sum_{n=1}^{M-1} a_{n} e^{-\mu_{n} s}$ is entire and $F_{M}$ can be continued anywhere $F$ can be, (7) will provide the analytic continuation of $G$ if we can continue $h(s, s)$ along paths which continue $F$.

Let $w \in \tilde{K}$ and choose any rectifiable path along which $F$ can be continued away from $\operatorname{Re} s>\sigma_{F}$. Let the path come from infinity along the real axis to $1+\sigma_{F}$ then to some $\tilde{s}$. Let $\Gamma$ be that portion of the path from $\sigma_{F}+1$ to $\tilde{s}$.

Now, $\Phi(w, u-s)$ is an entire function of $(w, s)$ and $F_{M}(u)$ is analytic in $\operatorname{Re} u>\sigma_{F}$ and recalling (5) we see that

$$
h_{1}(w, s) \equiv \int_{\sigma_{F}+1}^{\infty} \Phi(w, u-s) F_{M}(u) d u
$$

is an entire function of $s$ for each fixed $w \in \tilde{K}$ and an analytic function of $w$ in $\tilde{K}$ for each fixed $s \in \mathbf{C}$. Thus $h_{1}(w, s)$ is an analytic function of $(w, s)$ in $\tilde{K} \times \mathbf{C}$. Since $K$ is arbitrarily large, $h_{1}(s, s)$ is an entire function of $s$. Now 
there exists a number $r$ depending only on $\Gamma$ and $F_{M}$ such that $F_{M}$ is analytic in discs of radius $r$ centered at each point of $\Gamma$.

Suppose that

$$
h_{2}(w, s)=\int_{s}^{\sigma_{F}+1} \Phi(w, u-s) F_{M}(u) d u
$$

were not continuable up to $\tilde{s}$ along $\Gamma$ for some $w_{0} \in K$. Let $s_{1}$ be a point on $\Gamma$ between $\tilde{s}$ and $1+\sigma_{F}$ so that at every point on $\Gamma$ between $1+\sigma_{F}$ and $s_{1}$ the continuation exists but continuation past $s_{1}$ along $\Gamma$ is not possible. Choose $s_{0}$ on that part of $\Gamma$ connecting $s_{1}$ with $1+\sigma_{F}$ so that the length of the curve from $s_{0}$ to $s_{1}$ along $\Gamma$ is less than $r / 4$. See Figure 1 .

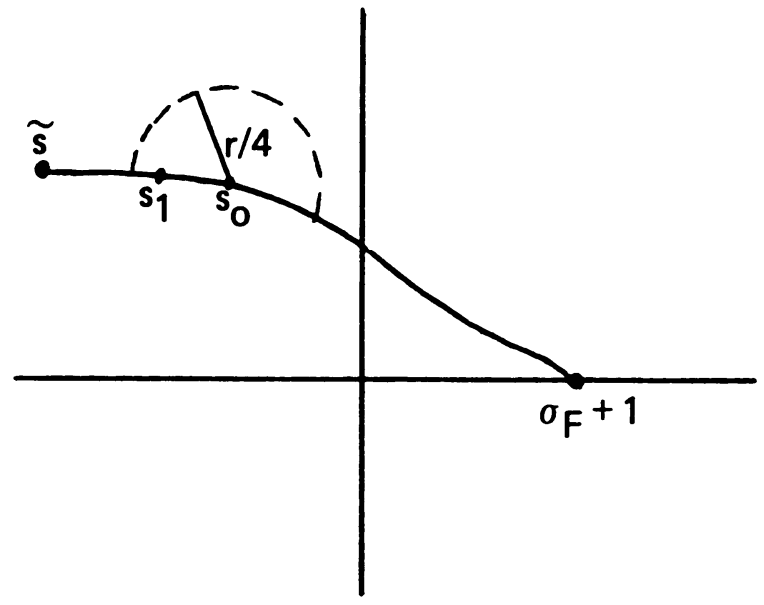

Figure 1

Now

$$
h_{2}(w, s)=\int_{s_{0}}^{1+\sigma_{F}} \Phi(w, u-s) F_{M}(u) d u+\int_{s}^{s_{0}} \Phi(w, u-s) F_{M}(u) d u .
$$

By an argument analogous to one given for $h_{1}$ one sees that

$$
h_{3}(w, s)=\int_{s_{0}}^{1+\sigma_{F}} \Phi(w, u-s) F_{M}(u) d u
$$

is a holomorphic function of $(w, s)$ in $\tilde{K} \times \mathbf{C}$. Since $K$ is arbitrary, $h_{3}(s, s)$ is entire in $s$. Now write

$$
\begin{aligned}
\int_{s}^{s_{0}} \Phi(w, u-s) F_{M}(u) d u \\
\quad=\int_{s_{0}}^{2 s_{0}-s} \Phi\left(w, v-s_{0}\right) F_{M}\left(v+s-s_{0}\right) d v \equiv \Psi(w, s) .
\end{aligned}
$$

Consider only the set of $s$ satisfying $\left|s-s_{0}\right| \leq r / 2$. Consider $F_{M}(v+z)$ as a holomorphic function of $z$ in $|z|<r / 2$ for any $v$ on the path of the second integral in (12). If $v$ is on that path then $v=u-s+s_{0}$ some $u$ on the path 
of the first integral in (12), that is on a portion of $\Gamma$. Then for $s, z$ as above and such a $v,|u-(v+z)|=\left|s-s_{0}-z\right|<\left|s-s_{0}\right|+|z|<r$ and $F_{M}$ is holomorphic in the disc of radius $r$ about $u$.

So one can write

$$
F_{M}(v+z)=\sum_{k=0}^{\infty} \frac{F_{M}^{(k)}(v)}{k !} z^{k}
$$

converging uniformly in $|z| \leqslant \frac{1}{2} r-\delta$ for all $\delta \in(0, r / 2)$. In particular,

$$
F_{M}\left(v+s-s_{0}\right)=\sum_{k=0}^{\infty} \frac{F_{M}^{(k)}(v)}{k !}\left(s-s_{0}\right)^{k}
$$

converges uniformly for $\left|s-s_{0}\right| \leqslant \frac{1}{2} r-\delta$ for $\delta \in(0, r / 2)$. Substituting (12) into (13) we obtain

$$
\Psi\left(w_{0}, s\right)=\sum_{k=0}^{\infty} \frac{\left(s-s_{0}\right)^{k}}{k !} \int_{s_{0}}^{2 s_{0}-s} \Phi\left(w_{0}, v-s_{0}\right) F_{M}^{(k)}(v) d v
$$

converging uniformly for $\left|s-s_{0}\right| \leqslant \frac{1}{2} r-\delta$. Now, $F_{M}^{k}(v)$ is holomorphic in the disc of radius $r$ about $s_{0}$ and for $v$ on the path of integration in (13),

$$
\left|v-s_{0}\right| \leqslant\left|2 s_{0}-s-s_{0}\right|=\left|s-s_{0}\right|<r .
$$

So $\int_{s_{0}}^{2 s_{0}-s} \Phi\left(w_{0}, v-s_{0}\right) F_{M}^{(k)}(v) d v$ is holomorphic as a function of $s$ on $\{s$ : $\left.\left|s-s_{0}\right|<r / 2\right\}$. Since the convergence in (14) is uniform one has that $\Psi\left(w_{0}, s\right)$ is analytic on $\left\{s:\left|s-s_{0}\right|<(r / 2)-\delta\right\}$. From (10), (11) and (12) then $h_{2}\left(w_{0}, s\right)$ is analytic on $\left\{s:\left|s-s_{0}\right|<(r / 2)-\delta\right\}$. But $\left|s_{1}-s_{0}\right|<r / 4<$ $(r / 2)-\delta$ for $\delta=r / 8$, so $h_{2}\left(w_{0}, s\right)$ is analytic at $\left(w_{0}, s_{1}\right)$, a contradiction.

So, for each $w \in \tilde{K}, h_{2}(w, s)$ represents an analytic function of $s$ along paths which continue $F$.

If $s$ is fixed at say $\hat{s}$ on the curve $\Gamma$, noting that $\Phi(w, u-\hat{s})$ is entire in all its variables and that $F_{M}(u)$ is analytic on the path of integration we see that

$$
h_{2}(w, \hat{s})=\int_{\hat{s}}^{\sigma_{F}+1} \Phi(w, u-\hat{s}) F_{M}(u) d u
$$

defines an analytic function of $w$ on $\tilde{K}$. Thus $h_{2}(w, s)$ is an analytic function of $(w, s)$ for $w \in \tilde{K}$ and $s$ on any path continuing $F$. Since $K$ is arbitrarily large we see that $h_{2}(s, s)$ can be continued anywhere $F(s)$ can be continued. Thus from (6), (8), (9) and the preceding arguments $h(s, s)=h_{1}(s, s)+$ $h_{2}(s, s)$ is continuable along paths continuing $F$. Thus

$$
G(s)=F_{M}(s)+\sum_{n=1}^{M-1} a_{n} e^{-\mu_{n} s}+h(s, s)
$$

can be continued along those paths as well. 


\section{REFERENCES}

1. L. Bieberbach, Analytische Fortsetzung, Springer, Berlin, 1955.

2. A. Ostrowaki, Uber die analytische Fortsetzung von Taylorschen und Dirichletschen Riehen, Math. Ann. 129 (1955), 1-43.

3. S. Mandelbrojt, Dirichlet series, D. Reidel Publishing Company, Dordrecht-Holland, 1972.

DePartment of Mathematics, UNIVERstTy OF Kentucky, LeXington, Kentucky 40506

Current address: Department of Mathematics, Wesleyan College, Macon, Georgia 31201 